TPI-MINN-01/56

UMN-TH-2037/01

\title{
Flavordynamics with Conformal Matter and Gauge Theories on Compact Hyperbolic Manifolds in Extra Dimensions
}

\author{
D. A. Demir and M. Shifman \\ Theoretical Physics Institute, University of Minnesota, Minneapolis, MN 55455
}

\begin{abstract}
We outline a toy model in which a unique mechanism may trigger a dynamical chain resulting in key low-energy regularities. The starting points are a negative cosmological term in the bulk and conformally invariant nongravity sector. These elements ensure compactification of the extra dimensional space on a compact hyperbolic manifold (with the negative and constant scalar curvature). The overall geometry is then $\mathbf{M}_{4} \times \mathbf{B}_{n}$. The negative curvature on $\mathbf{B}_{n}$ triggers the formation of the four-dimensional defect which provides in turn a dynamical localization of ordinary particles. It also leads, simultaneously, to a spontaneous breaking of gauge symmetry through a Higgs mechanism. Masses of the fermions, gauge bosons and scalars all derive from the curvature of the internal manifold such that the Higgs boson is generally heavier than the gauge bosons. The factorizable geometry $\mathbf{M}_{4} \times \mathbf{B}_{n}$ and flatness of $\mathbf{M}_{4}$ require fine-tuning.
\end{abstract}




\section{Introduction}

Theories with large extra spatial dimensions have allowed one to reformulate the hierarchy problem in a geometric paradigm [1, 2, 3]. If space-time is in fact $(4+n)$ dimensional the Planck scale of gravity in four dimensions, $M_{\mathrm{Pl}}$, is determined by the fundamental $(4+n)$-dimensional scale, $M_{\star}$, and geometry of the extra space. In the simplest case, when the space-time is a product of the four-dimensional Minkowskian space-time $\mathbf{M}_{4}$ and an $n$-dimensional compact space $\mathbf{B}_{n}$, the two gravitational scales are related as follows:

$$
M_{\mathrm{Pl}}^{2}=\mathcal{V}_{n} M_{\star}^{n+2}
$$

where $\mathcal{V}_{n}$ is the volume of $\mathbf{B}_{n}$. If it is large enough, the fundamental gravity scale $M_{\star}$ may be as low as $\sim 1 \mathrm{TeV}$ [1], [3].

A key element of such higher-dimensional scenarios is the localization of matter on stable topological defects (branes [4], domain walls [5, [6] or vortices [1]; see also a recent discussion [7]) embedded in $(4+n)$-dimensional bulk, with thickness $\lesssim M_{\star}^{-1}$ and surface tension $T \gtrsim M_{\star}^{4}$.

This paradigm offers a wealth of novel explanations for the observed phenomenology - patterns of supersymmetry and electroweak symmetry breaking [8], threegenerational structure [9], ultra-light neutrinos [10], proton stability [11], etc. It turns out that each particular basic aspect of phenomenology explored so far is compatible with the brane-world ideas. Such a strategy - confronting established phenomenology with the brane-world ideas one by one - seems reasonable at the present, exploratory stage. The aspect which we address in this paper is a proliferation of distinct scales and mechanisms in the current brane-world scenarios. As simple brane-world ideas, that had been put forward several years ago, were progressing and developing, they incorporated contrived "sub-mechanisms" and substructures, so that now there is an apparent menace of producing a "personal" model for each particular phenomenon. We pose a question whether it is possible to find economic ways by combining several seemingly distinct mechanisms into one. More concretely, we assume that at the primary stage the matter sector of the theory has no mass parameters whatsoever (i.e. purely conformal); the only mass parameters enters through gravity. It then triggers a domain wall formation (determining its size and tension), and electroweak symmetry breaking.

Conformal invariance - the invariance of the physical laws under rescalings of all lengths and durations by a common factor (see e.g. [12, 13]) - is broken in nature by particles' masses. If one starts from conformal matter, as we do, it is concievable that mass parameters can penetrate from gravity in two distinct ways. First, gravity loops generate, generally speaking, dimensionful constants in operators appearing in the Lagrangian for the matter sector. This effect is not the one we are interested in. We will ignore gravity loop corrections altogether. This is a rather arbitrary assumption since we cannot indicate a dynamical pattern ensuring the required suppression of the gravity loops. Being aware that this is a weak point we will, nevertheless, accept this assumption (quantum gravity is not a complete 
theory, anyway), and will concentrate on another option. Treating gravity at the classical level, we will ask the question:

- Is it possible that the explicit breaking of conformal symmetry in the gravity sector (due to the bulk cosmological constant $\Lambda$ and other possible sources) induces the spontaneous breakdown of the conformal, gauge and other symmetries in the matter sector in an empirically viable way?

Certainly, such a scenario does not make any sense in the context of the fourdimensional theories, since in this case the fundamental gravity scale is given by $M_{\mathrm{Pl}} \sim 10^{19} \mathrm{GeV}$, while, say, the electroweak scale is $\sim 10^{2} \mathrm{GeV}$. However, in the context of the brane world scenarios, with low gravitational scales in the ballpark of $M_{\star} \sim 10^{3} \mathrm{GeV}$, the question above does not seem absurd. One may suspect that the mass scales generated in the matter sector will be of the same order of magnitude as the higher-dimensional fundamental scale $M_{\star}$.

We will consider $n$ codimensions and discuss dynamics of a conformally-invariant gauge theory on a factorizable manifold $\mathbf{M}_{4} \times \mathbf{B}_{n}$, where the first factor represents our four-dimensional space-time, while $\mathbf{B}_{n}$ represents extra dimensions and is compact. It will be arranged that the curvature scalar on $\mathbf{B}_{n}$ will eventually trigger the spontaneous breakdown of all symmetries in the matter sector. There are three obvious requirements to be met. We must take care of: (i) the cosmological constant on $\mathbf{M}_{4}$; (ii) the apparent gravitational scale on $\mathbf{M}_{4}$, i.e. $M_{\mathrm{Pl}}$; (iii) the scale of the electroweak symmetry breaking, i.e. the weak gauge boson masses. These requirements determine the size and curvature of the internal manifold, as well as the bulk cosmological constant, in a correlated way.

Our construction bears an illustrative nature. As a reference point, we will keep in mind something like the Standard Model (SM). However, we will focus mainly on general aspects believing that developing particular details would be premature at this stage.

The basic elements are as follows. To study the electroweak symmetry breaking we will need to deal with a Higgs field. To ensure that the matter sector is described by an effective Lagrangian which is conformally invariant we will need to introduce a universal dilaton field which replaces all dimensionful SM parameters. Finally, we will need a "defect builder" $\phi(x)$ (responsible for the formation of the topological defect), on top of bulk gauge and fermion fields [12, 13.

There is a certain conceptual similarity between the model we suggest and the warped-compactification scenario of Randall and Sundrum [14]. In both cases the driving force is gravity. This similarity does not go beyond the conceptual level, however. In particular, the Randall-Sundrum model implies a single codimension, which is not the case in our model. The hierarcy of scales is totally different too.

The organization of the paper is as follows. In Sec. 2 we consider a conformallyinvariant gauge theory in the factorizable geometry $\mathbf{M}_{4} \times \mathbf{B}_{n}$. After specifiying the properties of $\mathbf{B}_{n}$ required in order to get appropriate phenomenology, we discuss the emergence of $M_{\mathrm{Pl}}$ form $M_{\star}$, the formation of the topologically stable defect and matter localization on the defect. In Sec. 3 we discuss stability of the factoriz- 
able geometry. In particular, we check the consistency of the static background by tuning the long-distance cosmological constant to zero while keeping the tension of order of $M_{\star}^{4}$. In Sec. 4 we summarize our conclusions and comment on similarities/distinctions with other popular brane-world scenarios.

\section{Conformal gauge theories on compact hyper- bolic manifolds}

The framework of our discussions is a $(4+n)$ dimensional static factorizable geometry $\mathbf{M}_{4} \times \mathbf{B}_{n}$ where $\mathbf{B}_{n}$ is a compact manifold and $\mathbf{M}_{4}$ is the ordinary (empirically flat) space-time. In the static limit, generically, the compact manifold can have positive (e.g. an $n$-sphere $\mathbf{S}_{n}$ ), vanishing (e.g. an $n$-torus $\mathbf{T}_{n}$ ), or negative (e.g. an $n$-dimensional compact hyperbolic space) curvature scalar, depending on its geometry and topology 15, 16. Compact negative scalar curvature manifolds can be obtained from a noncompact one by applying a known procedure, see below. It is also necessary to verify the stability of the chosen background geometry. This issue will be discussed in Sec. 3.

As was already mentioned, we will require the nongravity part of the theory of the SM type to be conformal in the $(4+n)$-dimensional space. The conformal invariance is achieved through the dilaton coupling. Then, the matter part of the stress tensor is strictly traceless 7 . The traceless nature of the stress tensor is particularly important for us since it implies that the curvature scalar, $\mathcal{R}$, is entirely determined from the classical gravity equations by the bulk cosmological term and the background geometry, independently of the matter sector dynamics.

In general, the conformal invariance puts severe restrictions on possible couplings of the matter fields [12, 13. One key aspect is that the matter sector can contain no mass parameters - they can be generated only via gravitational interactions. At tree level, the matter sector couples to gravity via the minimal coupling to the metric field, and via the conformal coupling $\mathcal{R} \sigma^{2}$ of a scalar field $\sigma$. It is this latter coupling that is particularly important as it induces mass terms for scalars in the constant-curvature background. Neglecting the curvature of $\mathbf{M}_{4}$ (as it will eventually be tuned to zero) one concludes that a conformal scalar is either massive $(\mathcal{R}>0)$, masless $(\mathcal{R}=0)$, or tachyonic $(\mathcal{R}<0)$ depending on the structure of $\mathbf{B}_{n}$.

Our goal is generation of the observed particle spectrum from a conformal higherdimensional gauge theory. It is clear then that the induced breaking of the conformal invariance in the matter sector must generate an instability in the vacuum state. Obviously, for this to happen, it is necessary to have a negative curvature scalar (this may not be sufficient, as will be discussed below).

In general, a smooth compact manifold $\mathbf{B}_{n}$ of constant negative curvature is obtained from the covering space $\mathbf{H}_{n}$ of $n$-dimensional hyperbolic spaces by modding

\footnotetext{
${ }^{1}$ Here we neglect possible conformal anomalies which are not expected to play a role; moreover, for odd $n$ (the case $n=3$ may be preferrable for our purposes) they are absent.
} 
out by a freely and discontinuously acting (with no fixed points) subgroup $\Gamma$ of its isometry group. Therefore, hereon we take the internal manifold to be $\mathbf{B}_{n}=\mathbf{H}_{n} / \Gamma$, with the constant negative curvature $\mathcal{R}_{0}$. This is a highly curved negative-curvature manifold with a global anistropy and rigidity (no massless shape moduli) [15, 19]. The volume of such manifolds grows exponentially with their linear size, and it is the largest linear extension $L$ that dominates

$$
\mathcal{V}_{n}=\left|\mathcal{R}_{0}\right|^{-n / 2} e^{(n-1) \sqrt{\left|\mathcal{R}_{0}\right|} L}
$$

in $n \geq 2$ codimensions. Here it is assumed that $\left|\mathcal{R}_{0}\right|^{1 / 2} L \gg 1$ and we neglected irrelevant angular factors in Eq. (2).

The graviton zero mode on such manifolds is a constant [15], and, therefore, the hierarchy problem is solved by virtue of their large volume. Combining Eqs. (1) and (2) we get

$$
\frac{M_{\mathrm{Pl}}^{2}}{M_{\star}^{2}}=e^{(n-1) \sqrt{\left|\mathcal{R}_{0}\right|} L}\left(\frac{M_{\star}^{2}}{\left|\mathcal{R}_{0}\right|}\right)^{n / 2} .
$$

A huge hierarchy between $M_{\star}$ and $M_{\mathrm{Pl}}$ is generated by the topological invariant $\exp \left[(n-1) \sqrt{\left|\mathcal{R}_{0}\right|} L\right]$ in $\mathcal{V}_{n}$. Since the dependence on $L$ is exponential, unlike in the original proposal [1, 3], one can settle for a microscopic size of the compact manifold in the extra dimensions. Clearly, the fundamental scale of gravity $M_{\star}$ does not need to exactly coincide with the scale of $\left|\mathcal{R}_{0}\right|$ (and also with the bulk cosmological constant $\Lambda$, see below). In fact, one can choose $\left|\mathcal{R}_{0}\right| \lesssim M_{\star}^{2} \sim(\mathrm{TeV})^{2}$ by adjusting $\sqrt{\left|\mathcal{R}_{0}\right|} L$ appropriately. For instance, if

$$
n=3, \quad M_{\star} \sim 1 \mathrm{TeV}, \quad \text { and } \quad\left|\mathcal{R}_{0}\right| \sim(0.5 \mathrm{TeV})^{2},
$$

the maximal linear extension of the manifold turns out to be

$$
L \approx 34\left|\mathcal{R}_{0}\right|^{-1 / 2} \approx 1.4 \times 10^{-15} \mathrm{~cm} .
$$

Unlike the Arkani-Hamed-Dimopoulos-Dvali (ADD) scenario [1] where the size of the extra dimensions is macroscopic (and is at the border of what is allowed by the current gravity experiments [17], $\sim 0.1 \mathrm{~mm}$ ) in the case at hand $L$ is microscopic.

\subsection{Relation between the scalar curvature and the bulk cos- mological term}

In a higher-dimensional theory whose nongravity part is strictly conformal, the Einstein equations imply that the curvature scalar is determined solely by the vacuum energy densities (the bulk cosmological constant plus other possible sources). For a factorizable geometry $\mathbf{M}_{4} \times \mathbf{B}_{n}$, assuming that $\mathbf{M}_{4}$ is already flattened thanks to appropriate source terms, the compact space possesses the curvature scalar $\mathcal{R}=$ 
$2 n \Lambda /(n-2)$ where $\Lambda$ is the bulk cosmological term. For grasping the importance of the static character of the internal manifold, one notices that the curvature scalar has the form

$$
\mathcal{R}=\mathcal{R}\left(d^{2} r / d t^{2},(d r / d t)^{2}, r^{2}\right),
$$

$r$ being the curvature radius of the internal manifold [16]. Clearly, for ensuring a static compact space, the intrinsic curvature contribution (the only piece independent of the time derivatives) to $\mathcal{R}$ must be balanced by the bulk cosmological term in the field equtions: $\mathcal{R} \propto \Lambda$.

In analyzing the matter sector, we take the factorizable static background geometry as the basic ansatz. The consistency of this assumption as well as the relation between the curvature scalar and the bulk cosmological term are best understood after reducing the bulk field theory to $\mathbf{M}_{4}$, and requiring the stability and vanishing of the long-distance (four-dimensional) cosmological term. Such details are deferred till Sec. 3.

\subsection{Destabilization of scalar fields}

It is convenient to discuss first the destabilization of the scalar potential of a typical scalar field. For instance, a dilaton $\sigma$ may be described by the Lagrangian

$$
\mathcal{L}[\mathcal{R}, \sigma]=(1 / 2)\left[G^{A B} \partial_{A} \sigma \partial_{B} \sigma-\zeta_{c} \mathcal{R}_{0} \sigma^{2}-\lambda_{\sigma} \sigma^{2 \gamma}\right]
$$

where $\sigma$ is a real field,

$$
\zeta_{c}=\frac{n+2}{4(n+3)} \quad \text { and } \quad \gamma=\frac{n+4}{n+2},
$$

as required by the conformal invariance [12, 13]. The potential of $\sigma$,

$$
V(\sigma)=\zeta_{c} \mathcal{R}_{0} \sigma^{2}+\lambda_{\sigma} \sigma^{2 \gamma}
$$

has twof critical points $\sigma_{\max }=0$ with $V\left(\sigma_{\max }\right)=0$, and

$$
\begin{aligned}
\sigma_{\min } & =\left(-\frac{\zeta_{c} \mathcal{R}_{0}}{\gamma \lambda_{\sigma}}\right)^{1 / 2(\gamma-1)} \\
V\left(\sigma_{\min }\right) & =\left(\frac{1-\gamma}{2 \gamma}\right)\left(\gamma \lambda_{\sigma}\right)^{-1 /(\gamma-1)}\left|\zeta_{c} \mathcal{R}_{0}\right|^{\gamma /(\gamma-1)},
\end{aligned}
$$

which correspond to local maximum and minimum, respectively. The small perturbations $\bar{\sigma}$ around these critical points have masses $m_{\sigma}^{2}(\max )=\zeta_{c} \mathcal{R}$ and $m_{\sigma}^{2}(\min )=$ $2(1-\gamma) \zeta_{c} \mathcal{R}_{0}$. Remember that the quantities $\mathcal{R}_{0}$ and $1-\gamma$ are negative.

The $\sigma$ quanta evolve in time as $\bar{\sigma} \sim e^{i m_{\sigma} t}$ which implies that small perturbations around $\sigma_{\max }=0$ are unstable. Since $\mathbf{B}_{n}$ is a compact manifold, one always has a

\footnotetext{
${ }^{2} \mathrm{~A}$ minimum at negative $\sigma$ is irrelevant for our purposes.
} 
zero mode solution $\bar{\sigma}(x, y)=$ const $\bar{\sigma}_{0}(x)$ where $y$ stands for extra coordinates, and $\bar{\sigma}_{0}(x)$ obeys the equation

$$
\square_{4} \bar{\sigma}_{0}(x)+\zeta \mathcal{R}_{0} \bar{\sigma}_{0}(x)=0
$$

whose solution is always destabilized. Note that a perturbative stability analysis of Ref. [18 referring to noncompact anti-de Sitter spaces is inapplicable in the case at hand due to the compactness of $\mathbf{B}_{n}$. The vacuum expectation value of $\sigma$ is necessarily nonvanishing for a negatively-curved internal manifold. This is a spontaneous breaking effect which will communicate the explicit conformal symmetry breaking of the gravity sector to the matter sector.

\section{3 "Defect builder" and the Higgs fields}

Having discussed the destabilization of a typical scalar field via constant negative curvature scalar, we now turn to the issue of localization of matter at distances $\lesssim M_{\star}^{-1}$ on (empirically flat) submanifold $\mathbf{M}_{4}$. (The bulk theory also possesses gauge and other symmetries, to be spontaneously broken; this will be discussed later). There are various field-theoretic and stringy mechanisms for localizing matter on $\mathbf{M}_{4}$. For our illustrative purposes we will utilize a field-theoretic framework put forward and developed in [5, 6] in which the ordinary four-dimensional space-time is a topologically stable defect. This is by no means a unique option. One could consider other known mechanisms leading to matter localization.

In general, the formation of the stable defect requires a spontaneously broken global symmetry. Moreover, the type of the defect depends on the number of extra dimensions: a domain wall in one codimension, a vortex line in two codimensions, and so on.

Unlike the spherical or toroidal structures [1], the manifold $\mathbf{B}_{n}$ under consideration is globally anistropic [19]; for solving the hierarchy problem only the largest linear size $L$ is relevant [15]. Therefore, as an approximate but physical picture, one can imagine $\mathbf{B}_{n}$ extending along a particular direction, say $y$, like a stick 3 of length $L$ and thickness $\delta \ll L$,

$$
\delta \sim\left|\mathcal{R}_{0}\right|^{-1 / 2}, \quad L / \delta \gg 1
$$

In other words, out of all $n$ dimensions, one is significantly larger than the remaining $n-1$. The latter, though needed to keep the curvature scalar negative and constant, are much smaller. Clearly, within such a picture, the dependence of matter fields on these $n-1$ dimensions can be neglected (as well as the corresponding components of, say, vector fields). The problem becomes effectively one-dimensional. In such quasi one-dimensional setting, the defect builder field $\phi$ can form a domain wall

3 The shape and size of the manifold depends on what subgroup of the isometry group of $\mathbf{H}_{n}$ is acting. For a detailed numerical study of $\mathbf{B}_{4}$ see [19. 
and dynamically localize the matter f. Consequently, the scalar sector, composed of the defect builder $\phi$, dilaton $\sigma$ and the Higgs field $H$, may be described by the Lagrangian

$$
\begin{aligned}
\mathcal{L}[\mathcal{R}, \phi, \sigma, H] & =(1 / 2)\left[G^{A B} \partial_{A} \phi \partial_{B} \phi-\zeta_{c} \mathcal{R} \phi^{2}-\lambda_{\phi} \phi^{4} \sigma^{2(\gamma-2)}\right]+ \\
& G^{A B}\left(\mathcal{D}_{A} H\right)^{\dagger} \mathcal{D}_{B} H-\left(\zeta_{c} \mathcal{R}+\lambda_{0} \phi^{2} \sigma^{2(\gamma-2)}\right) H^{\dagger} H-\lambda_{h}\left(H^{\dagger} H\right)^{\gamma}
\end{aligned}
$$

to which (4) is to be added. Here we take $\lambda_{\phi}$ to be sufficiently small compared to $\lambda_{\sigma}$ so that the change in $\sigma_{\min }$ is small. For simplicity one can assume $\phi$ to be real. The interactions of the scalars are such that there is a manifest $\mathcal{Z}_{2}$ invariance under which $\phi \rightarrow-\phi, \sigma \rightarrow \sigma$, and $H \rightarrow H$.

In Eq. (7) we dropped several terms allowed by symmetries as such terms are not essential for the mechanism discussed here. For instance, in the Higgs interaction one can add terms $\phi^{4} \sigma^{2(\gamma-3)} H^{\dagger} H$ and $\sigma^{2(\gamma-1)} H^{\dagger} H$, whose main effect would be to split the scalar masses. In any case, as we are not aiming at reproducing the exact electroweak spectrum, such details are not essential.

In the $\sigma=\sigma_{\min }$ background, the potential of $\phi$ is destabilized, leading to a spontaneous breakdown of the $\mathcal{Z}_{2}$ symmetry with two possible VEVs,

$$
\phi_{0}= \pm \varphi_{0}, \quad \varphi_{0}=\left(\frac{\left|\zeta_{c} \mathcal{R}_{0}\right|}{2 \lambda_{\phi} \sigma_{\min }^{2(\gamma-2)}}\right)^{1 / 2}
$$

This allows one to build a wall with the profile

$$
\phi(y)=\varphi_{0} \tanh \left(m_{\phi} y\right)
$$

interpolating between $-\varphi_{0}$ and $+\varphi_{0}$ as $y$ changes from $-L / 2$ to $L / 2$. Here $m_{\phi}^{2}=$ $\left|\zeta_{c} \mathcal{R}_{0}\right|$ is the mass of the $\phi$ quantum. Needless to say that $m_{\phi}$ is assumed to be large, $m_{\phi} L \gg 1$. Then the wall thickness is much less than $L$. The inverse thickness of the wall as well as its tension $\left(T \sim\left|\zeta_{c} \mathcal{R}_{0}\right|^{2}\right)$ are in the ballpark of $M_{\star}$ to the appropriate power (see Fig. 1).

We now turn to the discussion of the Higgs field in the domain wall background. With $\lambda_{0}>0$ the term $\phi^{2} \sigma^{2(\gamma-2)} H^{\dagger} H$ induces a positive potential for $H$ in the bulk, while in the core of the defect the potential vanishes. Then the Higgs field is stable outside the wall, while a tachyonic mass develops inside the wall, and, hence, a nonvanishing VEV of the Higgs field develops inside the wall Ð. More concretely, away from the wall, in the bulk, the wall builder attains one of its two vacuum values, and the effective (mass) ${ }^{2}$ of the Higgs field is

$$
\widetilde{m}_{H}^{2}=\left\{-1+\lambda_{0} /\left(2 \lambda_{\phi}\right)\right\}\left|\zeta_{c} \mathcal{R}_{0}\right| \text {. }
$$

\footnotetext{
4 The topological stability of the domain wall requires an infinite extension for $y$, and therefore, the picture discussed here is approximate; the wall will be approximately stable. Its decay rate will be suppressed exponentially as $\exp \left(-\operatorname{const} L\left|\mathcal{R}_{0}\right|^{-1 / 2}\right)$.

${ }^{5}$ The impact of the gauge interactions of the Higgs field on stabilization/destabilization of the Higgs potential will be discussed in Sect. 2.4. It does not change the overall picture.
} 
The mass term (10) is positive provided $\lambda_{0}>2 \lambda_{\phi}$, and, consequently, the gauge symmetry remains unbroken in the bulk. In the core of the wall

$$
\widetilde{m}_{H}^{2} \approx-\left|\zeta_{c} \mathcal{R}_{0}\right|
$$

and the gauge symmetry is broken provided $\lambda_{0}>2 \lambda_{\phi}$. The constraint $\lambda_{0}>2 \lambda_{\phi}$ is a mild tuning, and such a choice does not produce any harm on the mechanism of the wall formation.

In the core of the domain wall, however, $\phi(y) \sim 0$, and thus, the Higgs field necessarily develops a nonvanishing $\mathrm{VEV}$

$$
|H|_{0}=\left(\frac{\zeta_{c}\left|\mathcal{R}_{0}\right|}{\gamma \lambda_{h}}\right)^{1 / 2(\gamma-1)}
$$

which leads, in turn, to a spontaneous breakdown of the gauge symmetry. In this minimum, the mass of the Higgs quantum is given by $m_{h}^{2}=4(\gamma-1)\left|\zeta_{c} \mathcal{R}_{0}\right|$. As mentioned above, had we included terms like $\phi^{4} \sigma^{2(\gamma-3)}$ and $\sigma^{2(\gamma-1)}$ the appearent degeneracy between $\sigma$ and $H$ quanta would be lifted, and the condition on $\lambda_{0}$ to avoid breaking of the gauge symmetry in the bulk would be also modified accordingly.

\subsection{Gauge fields}

To outline the gauge field dynamics let us consider an $\mathrm{SU}(2)$ gauge theory in the bulk with

$$
\mathcal{D}_{A} H=\left(\partial_{A}+i\left(g_{2} / 2\right) \tilde{\sigma} \vec{\sigma} \cdot \vec{W}_{A}\right) H
$$

where $\vec{W}_{A}$ are the three gluon fields with $4+n$ components, and $\widetilde{\sigma}$ is a dilaton with mass dimension $-n / 2$,

$$
\widetilde{\sigma}=-\left(1+\frac{2}{n}\right) \sigma^{-n /(n+2)}
$$

For the stick-like manifold configuration under consideration, $\vec{W}_{A}$ is effectively a five-dimensional gauge field. Then, in the core of the wall, the $\mathrm{SU}(2)$ symmetry is completely broken giving three massive vector bosons,

$$
M_{W}^{2}=\left(\frac{g_{2}}{\gamma-2}\right)^{2}\left(\frac{\lambda_{\sigma}}{\lambda_{h}}\right)^{1 /(\gamma-1)} \frac{\left|\zeta_{c} \mathcal{R}_{0}\right|}{\gamma \lambda_{\sigma}}
$$

whose degeneracy can be lifted by additional group factors (e.g. the hypercharge group $\left.\mathrm{U}(1)_{Y}\right)$. Outside the core of the wall the Higgs field does not condense, and the gauge theory remains in the non-Abelian phase. One can try to exploit this fact in order to use the mechanism for the gauge field localization on the wall suggested in Ref. [6]. 
The mechanism [6] requires that in the bulk we have an unbroken non-Abelian gauge theory, which develops string-like flux tubes with a nonvanishing string tension. Then, just like in the Meissner effect (where a perfect superconductor repels the magnetic field), the "superconducting" bulk (where the wall-builder condenses keeping the gauge invariance exact) will repel the flux tubes of the electric field confining them to the core of the topological defect.

The question whether or not the flux tubes are formed and the non-Abelian theories confine in $4+n$ dimensions is not completely clear. We do know that they confine in two, three and four dimensions. Moreover, at sufficiently strong coupling confinement persists in higher dimensions, as follows from lattices and from ADS/CFT-based arguments [20] (see also [1] for similar field-theoretic arguments). In the D-brane/string theory it is explicit. Thus, it is likely that in a certain range of the coupling constants $\lambda$ the effective coupling constant $\widetilde{\sigma} g$ is such that gaugenonsinglet objects are confined in the bulk at $n>0$. Then the mechanism of Ref. [6] applies. A particular model of how this mechanism localizes gluons was considered in Ref. [7] in detail. The problem is whether or not one can get a sufficiently small gauge coupling (compatible with phenomenology) inside the wall, where the gauge symmetry is spontaneously broken, and we are in the Higgs phase. To this end one can try to play with the variations of $\widetilde{\sigma}$ inside and outside the wall. This task would require a numerical analysis, which we postpone for the future.

In Sect. 2.3 we have discussed the bulk stabilization (core destabilization) of the Higgs potential with the gauge interactions switched off (see Eqs. (10) and (11)). Here we will argue that the phenomenon remains valid with the gauge interactions switched on. The gauge interactions induce a contribution to the Higgs mass term of the form

$$
\left\langle H^{\dagger}\left(-D_{\text {Euclid }}^{2}\right) H\right\rangle \rightarrow C M_{*}^{2} H^{\dagger} H
$$

where $D_{\text {Euclid }}^{2}$ is the Euclidean covariant Lapacian, and $C$ is a dimensioneless constant which depends on various other dimensionless constants in the theory, such as $\lambda_{\sigma}$. It is important that because of positivity of $-\left(D_{\text {Euclid }}^{2}\right)$, the constant $C$ is positive. It adds to the stabilizing term in the Higgs potential. If $C$ is adjusted to be not too large, the previous conclusion of the bulk stabilization and core destabilization remains intact.

The above dynamical trapping is not special to gauge fields. In fact, the bulk fermions will be localized on the wall too due to the confining gauge dynamics outside. For the purpose of localization, they may or may not be directly coupled to the wall builder as in [5, 21]). As a simple example, consider an $\mathrm{SU}(2)$ doublet $\psi$ and an $\mathrm{SU}(2)$ singlet $\psi^{\prime}$ with the Yukawa interaction

$$
\mathcal{L}_{Y}=y_{\psi} \tilde{\sigma} \bar{\psi} H \psi^{\prime}+\text { h. c. }
$$

Then in the core of the wall, where the $\mathrm{SU}(2)$ symmetry is spontaneously broken, there arises a massive fermion with mass $m_{\psi}=y_{\psi} M_{W} / \sqrt{g_{2}}$. To see how realistic 
this mass spectrum is, we take $n=3, \lambda_{\sigma} \sim \lambda_{h} \sim 1 / \gamma \ll 1$ and $\left|\mathcal{R}_{0}\right| \sim(350 \mathrm{GeV})^{2}$. This then gives $M_{W} \sim 100 \mathrm{GeV}, m_{\psi} \sim y_{\psi} M_{W}$, and $m_{h}=200 \mathrm{GeV}$ where one particularly notices that the Higgs boson is always heavier than the gauge bosons.

An important point to be addressed here is the chirality of the localized fermions. In general, fermions localized via the confining bulk gauge dynamics are not chiral. Moreover, in odd dimensions ( $n$ is odd) there is no chirality. In general [5, 6], a way out is provided by the defect-builder itself: if the bulk fermions are directly coupled to $\phi$, the zero modes are chiral, and their chirality is correlated with the topological charge of the wall. Explicitly, the bulk fermions $f$ and $f_{c}$ can be coupled as $y \widetilde{\sigma} \phi \bar{f} f+y_{c} \widetilde{\sigma} \phi \overline{f_{c}} f_{c}$ which deposit the chiral zero modes $f+\bar{f}^{\dagger}$ and $f_{c}^{\dagger}+\overline{f_{c}}$ whose couplings to the Higgs field as in (16) produces acceptable fermion masses in the core of the defect [1].

Concerning the gauge symmetry in the bulk, one should take care of gauge singlets, as $\psi^{\prime}$ in Eq. (16). To this end one may embed the SM gauge group in a larger non-Abelian one, e.g. the Pati-Salam group $\mathrm{SU}(4) \times \mathrm{SU}(2)_{L} \times \mathrm{SU}(2)_{R}$. Then using appropriate (in number and representation) Higgs fields one can obtain the SM spectrum below $M_{\star}$. In fact a realistic model with Pati-Salam group in $n=2$ codimensions have already been discussed in [1] where the defect builder forms a vortex line the throat of which consistently localizes the matter on $\mathbf{M}_{4}$. One notices that once the scalar sector is destabilized, the hierarchy needed among VEVs of various Higgs fields can be generated via their interactions by mild tuning of the parameters (e.g. couplings of the form $\phi^{4} \sigma^{2(\gamma-3)} H^{\dagger} H$ ).

\section{Stabilization of the factorizable geometry and flatness of $\mathrm{M}_{4}$}

The discussion in Sec. 2 was based on the factorizable background geometry $\mathbf{M}_{4} \times$ $\mathbf{B}_{n}$ where $\mathbf{B}_{n}$ is a static compact negative-curvature manifold. Here our primary concern is the stabilization of the extra dimensions. Let us recall, for instance, that in spherical or toroidal geometries [1, 16, 22] one has to stabilize the large extra dimensions against expansion, which requires the bulk cosmological constant be balanced with the curvature of the manifold, and against contraction, which requires, generally speaking, either brane-lattice crystallization or a topological invariant, e.g. Ramond-Ramond gauge field on $\mathbf{S}^{2}$ topology.

For the manifold structure under consideration, the main problem is to prevent the expansion of the internal manifold as its size is already required to be around the fundamental scale of gravity. As in positive- or zero-curvature spaces [16, 22] the stabilization against the expansion requires fine-tuning the bulk cosmological constant against the curvature term. Indeed, the analysis of [15] shows that a factorizable geometry of the form $\mathbf{M}_{4} \times \mathbf{B}_{n}$ almost automatically arises once the bulk cosmological constant is appropriately tuned.

The compact hyperbolic manifolds possess the important property of rigidity - 
their volume in units of $|\mathcal{R}|$ cannot be changed while maintaining the homogeneity of the space. Therefore, stabilization of the static factorizable background reduces to the stabilization of the curvature length $|\mathcal{R}|^{-1 / 2}$ of $\mathbf{B}_{n}$. The relevant part of the reduced bulk action in the far infrared is nothing but the long-distance (fourdimensional) cosmological constant,

$$
\Lambda_{4}(\mathcal{R})=\mathcal{V}_{n}\left(-M_{\star}^{n+2}(\mathcal{R}-2 \Lambda)+V\left(\sigma_{\text {min }}\right)\right)+\mathcal{K}(\mathcal{R})+T
$$

where $T>0$ is the wall tension (including the contribution of the Higgs potential). Here $\mathcal{K}(\mathcal{R})$ collectively denotes the contribution of the kinetic terms of the bulk scalar and vector fields [16, 22], and can be expanded as $\sum_{a>0} C_{a}|\mathcal{R}|^{a / 2} M_{\star}^{4-a}$ [15]. The stability of the factorizable configuration requires that

$$
\Lambda_{4}^{\prime}\left(\mathcal{R}=\mathcal{R}_{0}\right)=0 \quad \text { and } \quad \Lambda_{4}^{\prime \prime}\left(\mathcal{R}=\mathcal{R}_{0}\right)>0,
$$

together with the empirical requirement of $\Lambda_{4}\left(\mathcal{R}=\mathcal{R}_{0}\right)=0$. A straightforward calculation, which is particularly simple for large $n$, suggests that $\Lambda<0$ and $\mathcal{R} \equiv$ $\mathcal{R}_{0} \sim 2 \Lambda$. Moreover, $\Lambda_{4}^{\prime \prime}\left(\mathcal{R}_{0}\right)$ determines the masses of small fluctuations around $\mathcal{R}=\mathcal{R}_{0}$ to be $\mathcal{O}\left(M_{\star}\right)$ which is large enough to evade cosmological problems [15] with a light radion occuring in spherical and toroidal geometries [16. One notices that the bulk cosmological constant prevents the internal manifold from expanding indefinitely.

An important issue in the far infrared is the vanishing of the long-distance cosmological constant. Empirically, it is known that such a cancellation can be achieved by tuning the coefficients $C_{a}$ against the first and third terms in (17) which involves an extreme fine-tuning of the parameters - the cosmological constant problem. Clearly, one should excercise care in fine-tuning $\Lambda_{4}(\mathcal{R})$ to zero in order to make $\mathbf{M}_{4}$ flat, as it can result in a solution with $T \sim \mathcal{V}_{n} M_{\star}^{n+4} \gg M_{\star}^{4}=M_{\mathrm{Pl}}^{2} M_{\star}^{2}$ which is completely unnatural given the characteristic scale of the SM. Moreover, such a huge wall tension will destroy the initial ansatz on the background geometry. On the contrary, if $T \sim M_{\star}^{4}$, the back reaction on the wall on the solution under consideration is negligible, and our step-by-step strategy is justified.

One can estimate the back reaction of the wall by examining the Einstein equations. In the presence of the wall a new term in the right-hand side appears, proportional to $T \delta^{n}(\vec{y})$. In a rough approximation we will replace $T \delta^{n}(\vec{y})$ by $T / \mathcal{V}_{n}$, smearing the delta function homogeneously over the extra space. This will presumably lead to an overestimate of the back reaction. Neglecting irrelevant numerical factors, we get

$$
\Delta \mathcal{R} \sim \frac{T}{M_{\mathrm{Pl}}^{2}} .
$$

Let us remind the reader that this is a purely classical estimate, with all quantum corrections discarded. Moreover, $\Delta \mathcal{R}$ need not be constant, it depends on the profile of the wall solution in $\vec{y}$. It is clear that when $T \sim \mathcal{V}_{n} M_{\star}^{n+4}=M_{\mathrm{Pl}}^{2} M_{\star}^{2}$ the change in 


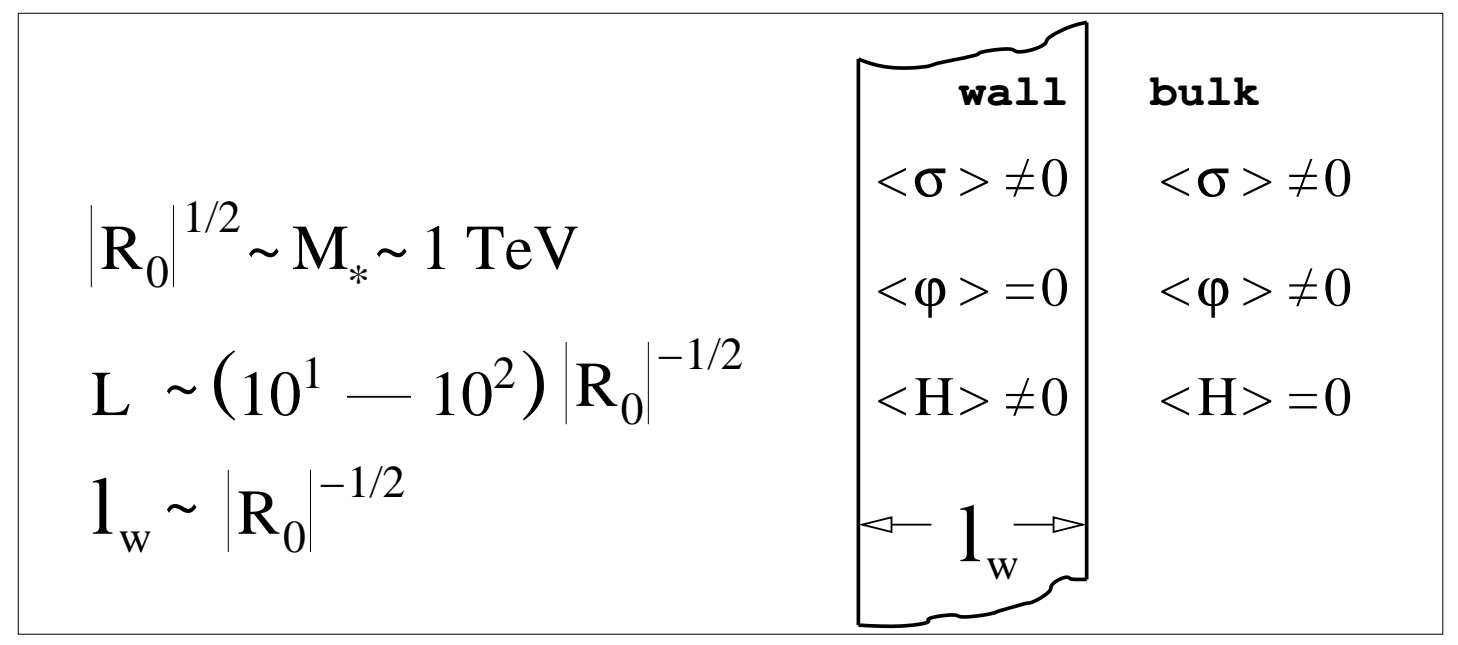

Figure 1: List of scales in the problem together with the VEVs of fundamental scalars in the bulk and the wall.

$\mathcal{R}_{0}$ is $\mathcal{O}(1)$, that is, the original ansatz on the curvature of the space is completely destroyed, and our construction collapses. Therefore, to prevent the ambient geometry from being significantly modified by the back reaction of the wall, one must require $T \lesssim M_{\star}^{4}$. This constraint represents a nontrivial aspect of the long-distance cosmological constant problem [16, 22, 15].

\section{Summary and Discussion}

Our basic starting point is the assumption that large compact extra dimensions exist and, as a result, the fundamental gravity scale $M_{\star} \sim \mathrm{TeV}$. Unlike the original suggestion [1] where most attention is paid to flat extra dimension, a (constant) negative curvature of the extra space is absolutely essential for the mechanism that we discuss. It is maintained, in turn, by a bulk negative cosmological term.

In this paper we outline a single-origin step-by-step mechanism which might be relevant for the low-energy phenomenology. Initially, the mass parameters are separated - the nongravity sector is assumed to be conformal. A (negative) cosmological term in the bulk ensures the existence of the negative curvature internal compact manifold. Its scalar curvature is related to the bulk cosmological term. It triggers then the formation of a topological defect, which, in turn, captures the SM matter fields. Simultaneously, it destabilizes the Higgs potential and triggers the spontaneous breaking of the gauge symmetry on the the defect (but not in the bulk). The scales of the problem and VEVs of the fundamental scalars are summarized in Fig 1. Clearly, all mass and length scales are fixed by the curvature scalar $\mathcal{R}_{0}$. 
The stability of the background geometry is consistent and self-sustaining provided that the bulk cosmological constant $\sim-M_{\star}^{2}$. The scale of gravity $M_{\star}$, the curvature scalar $\mathcal{R}_{0}$, bulk cosmological term $\Lambda$, and the largest linear extension of the compact manifold $L$ are the mass parameters of the model. These mass scales are interrelated via the requirements of (i) generating the correct electroweak spectrum $\left(\left|\mathcal{R}_{0}\right|^{1 / 2} \sim\right.$ Higgs mass); (ii) explaining enormity of $M_{\mathrm{Pl}}$ with respect to electroweak scale, and (iii) cancellation of the four-dimensional cosmological constant. A modest hierarchy is required between $L$ and $\left|\mathcal{R}_{0}\right|^{-1 / 2}$,

$$
L\left|\mathcal{R}_{0}\right|^{1 / 2} \gtrsim 30
$$

which may be purely numerical.

Recent studies of the cosmology of compact hyperbolic manifolds [23] suggest that the topology of extra space implies a very large entropy, and statistical averaging during collapse can account for the large scale flatness. However, in both publications [23] and [15] nongeometric degrees of freedom (in the bulk and on the defect) have not been included in the discussion. Analysis of several problems, e.g. $\mathrm{CP}$ violation, baryogenesis, inflation, structure formation etc., would be in order along the lines we have outlined in the present paper.

A toy $\mathrm{SU}(2)$ gauge model illustrates that the existence of a negatively-curved compact internal manifold alone is sufficient for breaking the symmetries of the matter sector so as to generate a flat topological defect trapping the massive as well as massless particle spectrum on it. Supersymmetry may or may not be needed. One can consider models with supersymmetry broken by the bulk cosmological term along these lines.

To conclude, it would be in order to compare the pattern suggested here with other popular brane-world scenarios [1, 14]. The compactness of the extra space is the basic feature of the ADD scenario. However, the characteristic size of the extra dimensions for compact hyperbolic structures is far below those obtained in spherical or toroidal geometries [1] (and orders of magnitude below the existing experimental bounds [17]). Small extra dimensions occur in the warped compactification scenario of Randall and Sundrum [14. The two approaches have similarities and distinctions. The basic conceptual similarity is the fact that in the both schemes gravity in the bulk plays a crucial role. The distinctions are technical but conspicuous. To name a few one notes that: $(i)$ the warped compactification scenarios work for a single extra dimension whereas the scenario discussed here requires at least two extra dimensions; ( $i i$ ) In the warped compactification scenarios five- and four-dimensional Planck scales essentially coincide while they are drastically different in the case of compact hyperbolic structures; (iii) In both scenarios the bulk cosmological constant is required to be negative, but its scale is different. As is seen from Fig. 1, the basic and practically the only scale in our model is $M_{*} \sim|\mathcal{R}|^{-1 / 2}$. In the Randal-Sundrum approach one starts from the $M_{\mathrm{Pl}}$ brane; (iv) The gravity-driven trigerring of the Higgs mechanism on the $\mathrm{TeV}$ brane is not a part of the Randal-Sundrum model per se, while in our model the core of the wall tends to destabilize the Higgs potential. 


\section{Acknowledgments}

We thank Gregory Gabadadze, A. Losev and Mikhail Voloshin for fruitful discussions on several aspects of this work. We also thank Elena Caceres and Nemanja Kaloper for useful communications. The work is supported in part by the US Department of Energy under the grant number DE-FG-02-94-ER-40823.

\section{References}

[1] N. Arkani-Hamed, S. Dimopoulos and G. R. Dvali, Phys. Lett. B 429, 263 (1998) hep-ph/9803315.

[2] I. Antoniadis, N. Arkani-Hamed, S. Dimopoulos and G. R. Dvali, Phys. Lett. B 436, 257 (1998) hep-ph/9804398.

[3] N. Arkani-Hamed, S. Dimopoulos and G. R. Dvali, Phys. Rev. D 59, 086004 (1999) hep-ph/9807344.

[4] J. Polchinski, Phys. Rev. Lett. 75, 4724 (1995) hep-th/9510017.

[5] V. A. Rubakov and M. E. Shaposhnikov, Phys. Lett. B 125, 136 (1983).

[6] G. R. Dvali and M. A. Shifman, Phys. Lett. B 396, 64 (1997) [Erratum-ibid. B 407, 452 (1997)] hep-th/9612128.

[7] S. L. Dubovsky and V. A. Rubakov, Int. J. Mod. Phys. A 16, 4331 (2001) hep-th/0105243.

[8] See e.g. the recent discussion: R. Barbieri, L. J. Hall and Y. Nomura, Phys. Rev. D 63, 105007 (2001) hep-ph/0011311.

[9] N. Arkani-Hamed and M. Schmaltz, Phys. Rev. D 61, 033005 (2000) hepph/9903417]; G. R. Dvali and M. A. Shifman, Phys. Lett. B 475, 295 (2000) hep-ph/0001072]; D. E. Kaplan and T. M. Tait, JHEP 0006, 020 (2000) hepph/0004200]; B. A. Dobrescu and E. Poppitz, Phys. Rev. Lett. 87, 031801 (2001) [hep-ph/0102010]; N. Borghini, Y. Gouverneur and M. H. Tytgat, hepph/0108094.

[10] G. R. Dvali and A. Y. Smirnov, Nucl. Phys. B 563, 63 (1999) hep-ph/9904211; N. Arkani-Hamed, S. Dimopoulos, G. R. Dvali and J. March-Russell, hepph/9811448.

[11] See e.g. A. Aranda and C. D. Carone, Phys. Rev. D 63, 075012 (2001) hep$\mathrm{ph} / 0012092$.

[12] H. Weyl, Space-Time-Matter (Dover, New York, 1952); R. H. Dicke, Phys. Rev. 125, 2163 (1962). 
[13] J. D. Bekenstein and A. Meisels, Phys. Rev. D 22, 1313 (1980).

[14] L. J. Randall and R. Sundrum, Phys. Rev. Lett. 83, 3370 (1999) hepph/9905221].

[15] N. Kaloper, J. March-Russell, G. D. Starkman and M. Trodden, Phys. Rev. Lett. 85, 928 (2000) [hep-ph/0002001.

[16] N. Arkani-Hamed, S. Dimopoulos and J. March-Russell, Phys. Rev. D 63, 064020 (2001) hep-th/9809124.

[17] C. D. Hoyle, U. Schmidt, B. R. Heckel, E. G. Adelberger, J. H. Gundlach, D. J. Kapner and H. E. Swanson, Phys. Rev. Lett. 86, 1418 (2001) hep ph/0011014.

[18] P. Breitenlohner and D. Z. Freedman, Phys. Lett. B 115, 197 (1982); Annals Phys. 144, 249 (1982); L. Mezincescu and P. K. Townsend, Annals Phys. 160, 406 (1985).

[19] N. Cornish and D. Spergel, math.DG/9906017; M. Trodden, hep-th/0010032;

G. D. Starkman, Class. Quant. Grav. 15, 2529 (1998).

[20] J. Polchinski and M. J. Strassler, Phys. Rev. Lett. 88, 031601 (2002) hepth/0109174].

[21] G. R. Dvali and M. A. Shifman, Nucl. Phys. B 504, 127 (1997) hepth/9611213].

[22] R. Sundrum, Phys. Rev. D 59, 085010 (1999) hep-ph/9807348; Phys. Rev. D 59, 085009 (1999) hep-ph/9805471.

[23] G. D. Starkman, D. Stojkovic and M. Trodden, Phys. Rev. Lett. 87, 231303 (2001) hep-th/0106143; Phys. Rev. D 63, 103511 (2001) hep-th/0012226. 